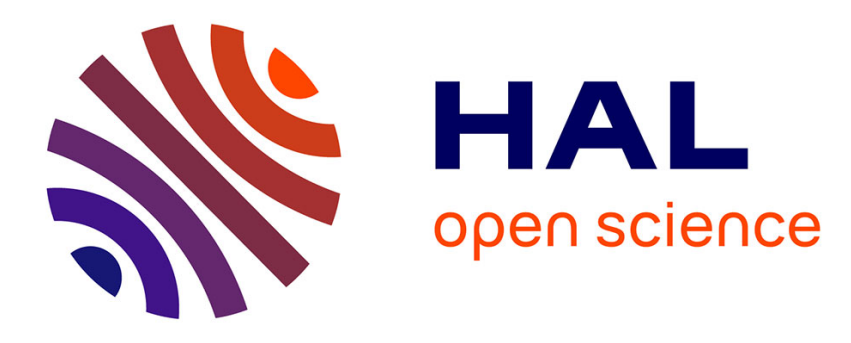

\title{
Cognition overrides orientation dependence in tactile viewpoint selection
}

Jessica Hartcher-O’brien, Malika Auvray

\section{To cite this version:}

Jessica Hartcher-O'brien, Malika Auvray. Cognition overrides orientation dependence in tactile viewpoint selection. Experimental Brain Research, 2016, pp.1-19. 10.1007/s00221-016-4596-6 . hal01286076

\section{HAL Id: hal-01286076 https://hal.sorbonne-universite.fr/hal-01286076}

Submitted on 10 Mar 2016

HAL is a multi-disciplinary open access archive for the deposit and dissemination of scientific research documents, whether they are published or not. The documents may come from teaching and research institutions in France or abroad, or from public or private research centers.
L'archive ouverte pluridisciplinaire HAL, est destinée au dépôt et à la diffusion de documents scientifiques de niveau recherche, publiés ou non, émanant des établissements d'enseignement et de recherche français ou étrangers, des laboratoires publics ou privés. 
Cognition overrides orientation dependence in tactile viewpoint selection

Jessica Hartcher-O’Brien ${ }^{1,2}$ and Malika Auvray ${ }^{1}$

1. Sorbonne Universités, UPMC Univ. Paris 06, UMR 7222, ISIR, F-75005, Paris, France

2. Institut Jean Nicod, CNRS, EHESS, Ecole Normale Supérieure, 29 Rue d'Ulm, 75005, Paris France.

Email: hartcher@isir.upmc.fr

\section{Acknowledgements}

JHO was funded by the Fyssen foundation. MA was funded by a grant from the Agence Nationale de la Recherche (ANR-11-JSH2-003-1). The Institut Jean Nicod laboratory receives financial support via the ANR-10-LABX-0087 IEC and ANR-10-IDEX-0001-02 PSL. 


\begin{abstract}
Humans are capable of extracting spatial information through their sense of touch: when someone strokes their hand, they can easily determine stroke direction without visual information. However, when it comes to the coordinate system used to assign the spatial relations to the stimulation, it remains poorly understood how the brain selects the appropriate system for passive touch. In the study reported here, we investigated whether hand orientation can determine coordinate assignment to ambiguous tactile patterns, whether observers can cognitively override any orientation-driven perspectives on touch, and whether the adaptation transfers across body surfaces. Our results demonstrated that the orientation of the hand in the vertical plane determines the perspective taken: An external perspective is adopted when the hand faces the observer, and a gaze-centred perspective is selected when the hand faces away. Participants were then adapted to a mirror-reverse perspective through training and the results revealed that this adapted perspective holds for the adapted surface, and generalizes to non-adapted surfaces, including across the body midline. These results reveal plasticity in perspective taking which relies on low-level postural cues (hand orientation) but also on higher order somatosensory processing that can override the low-level cues.
\end{abstract}

Keywords: Touch; viewpoint selection; ambiguous pattern of stimulation; coordinate assignment; frame of reference.

\title{
1. Introduction
}

Spatial information about the objects in our environment is accessible not only through vision, but also through our senses of audition and touch (Vallbo \& Johansson, 1984). For instance, people can interpret the direction of stimulation when a visual or an auditory stimulus is displayed moving from left to right. This also holds true in touch when a body part is stroke from left to right. The question of how left and right are assigned, and thereby of how spatial information is taken from the environment, depends on the sensor that is used (Newell, Ernst, Tjan, \& Bülthoff, 2001), with the mechanisms appearing to be more straightforward and established in vision than in touch.

In the case of visual viewpoint selection, when you watch your hand being stroked, the stroking pattern is initially coded in retinotopic coordinates. Depending on the task, visual information is then either maintained in retinotopic coordinates for immediate action or it is 
transformed into external coordinates so that the observer can maintain a stable perceptual experience despite eye movements, changes in the position of the observer relative to the object, and object perturbations (Holway \& Boring, 1941; Wexler \& Held, 2005).

In the case of touch, the brain's viewpoint on stimuli is similarly not arbitrary, i.e., when somebody strokes your hand, it is easy to distinguish whether they are stroking it from left to right or from right to left. However, unlike spatial processing in vision, at any given time there are potential contributions from multiple coordinate systems, even when the task and posture do not change (Parsons \& Shimojo, 1987; Volcic, Wijntjes, \& Kappers, 2009). For example, determining the stroke direction across the surface of the hand can initially be done by coding the information in hand-centred coordinates, where left and right are assigned according the spatial layout of the hand. However, the hand is not an independent system, but is always relative to the person's trunk, head, or eyes. In this case, the body-, cranio-, retino-topic, or gaze-centred (cranio- and retino-topic combined) coordinates can also be used to code and interpret the incoming tactile information. To summarize, concerning early stages of processing, visual information is initially coded in retinotopic coordinates and can then be transformed into external, craniotopic or motor coordinates depending on the task and goal of the observer. Whereas for touch several coordinate systems already compete at early stages of somatosensory processing (Heed, Buchholz, Engel, \& Röder, 2015).

Theoretically, for an optimal processing of passive touch, i.e., when no explicit action is required, the most parsimonious interpretation of the stroking of the hand is to not remap the information into another coordinate system. For the brain to do so would be unnecessarily costly; thus left and right would be assigned according to the hand's spatial layout. On the other hand, if one is actively engaged in the exploration of an object, then the incoming information may be most efficiently coded by transforming the incoming somatosensory information into external coordinates. By transforming the stimulation into external coordinates, the brain can maintain a stable representation of the object, despite distortions due to movement, and postural cues. However, the theoretical parsimonious approach of coding information in hand-centred coordinates is not necessarily what has been observed (Parsons \& Shimojo, 1987; Volcic et al., 2009). The question then becomes, what are the factors that determine this coordinate assignment process for passive touch?

Given the ability of the hand to explore objects from different vantage points, independent of the posture of the body, this surface may well exhibit unique reference frame selection relative to other body surfaces. Several studies approached the question of what perspectives may be taken on somatosensory stimuli on the hand (Azañón \& Soto-Faraco, 2008; Holmes, 2014; Prather \& Sathian, 2002; Volcic et al., 2009). In these studies, 
coordinate selection is based on tests that give changes in reaction time between the stimulus/hand placed in a baseline coronal position and when it changes to a different position (Prather \& Sathian, 2002). The increase in RT is an indication of the cost of changing reference frame (Volcic et al., 2009). Note, that the studies which behaviourally assess reference frame selection in passive touch (without motor activity or movement) on other body parts also used indirect methods such as RTs (Farrell \& Robertson, 1998; Schicke \& Röder, 2006; Torralbo, Santiago, \& Lupiáñez, 2006).

The exception, which provides a promising method to directly investigate viewpoint selection, was to use an ambiguous tactile symbol paradigm, which consists of grapheme stimuli (letters and numbers) drawn on the body surface. These graphemes potentially have several interpretations until the observer selects one coordinate system with which to interpret them. For instance, the letters 'b', 'd', 'p', and ' $q$ ' displayed on the skin are ambiguous until the observer takes a perspective and assigns coordinates to the cutaneous input, and consequently perceive one of the letters. Parsons and Shimojo (1987), for instance, used this paradigm and traced the graphemes on multiple body surfaces when the relative position and orientation of the surface was varied. When the experimenter traced the tactile patterns on the palm of the hand, stimuli were perceived relative to the position and orientation of the stimulated surface with respect to the body torso. Parson and Shimojo's results suggest the use of external frames of reference that are independent of the hand or head and that are associated with stimuli presented on the hand; that is, for Parsons \& Shimojo, according to the experimenter-defined coordinates. However, in Parsons and Shimojo's study (and in other studies using this paradigm on other body surfaces that the hand, e.g. (Corcoran, 1977)) the graphemes were always drawn manually by the experimenter. As a consequence, the transformation into experimenter-defined coordinates could be solely due to the influence of the experimenter himself biasing the participants' responses.

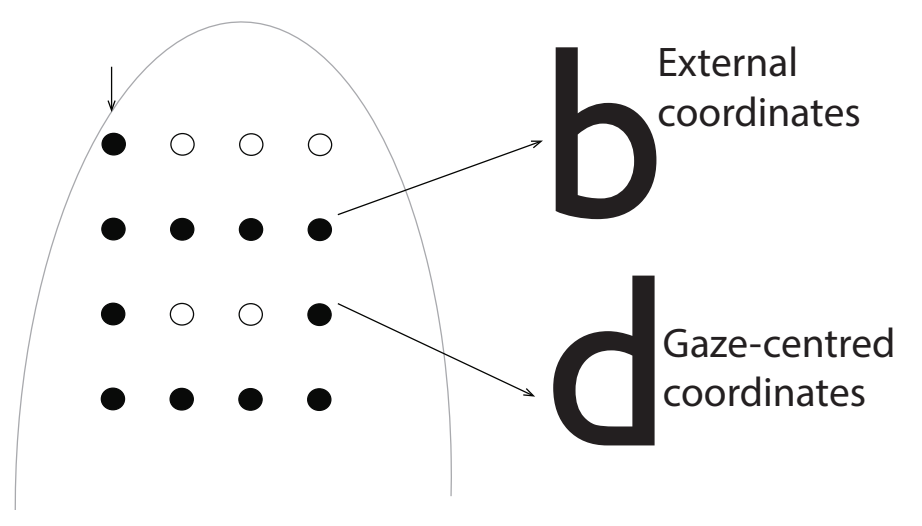

Figure 1. Cutaneous fingertip stimulation pattern and two potential interpretations depending on the reference frame selected. When the stimulation pattern ' $b$ ' is presented on the device surface (start-point and direction of stimulation indicated by the arrow), this pattern is interpreted as a 'b' if the spatial coordinates are assigned 
according to the external object's coordinates. However, this example pattern is ambiguous around the vertical axis with a mirror reversed perspective giving an interpretation of the pattern as the letter ' $d$ '. Here we define the object-centred coordinates as 'external', i.e. according to the spatial layout of the device surface. Given the position of the device in front of the observer, the mirror reverse of this assignment is consistent with cranio-topic, as well as retinoptopic coordinates. Because our task does not provide the means to disentangle the two potential coordinate systems we have adopted the description used by Harrar and Harris (2009) where they define a merged coordinate system of head and eye as 'gaze-centred'.

In our study, any experimenter-induced bias was removed from the equation by using a small braille pin display device to present the directional cutaneous patterns (see Figure 1). Our study was divided into 3 tasks. The first task investigated a similar question to Parsons and Shimojo's (1987) study: that is, whether the orientation of the hand could determine the mind's perspective on touch - however with our device and standardized procedure. However, in the case of our study, we were curious about interpretation of touch to the fingertip, not the palm (as investigated by Parsons \& Shimojo, 1987). We thus explored, for the first time, whether viewpoint selection could be overridden by adapting to the mirrorreverse perspective (in the second task). In a third task, we then measured whether this adapted perspective would generalise across non-adapted fingers and across the body midline.

\section{Methods}

2.1 Participants. Fourteen naïve participants (nine males) took part in the study. The average age was 26.8 years. The participants provided their written consent prior to the commencement of the experiment and were given $€ 8$ for their participation. The experiment took approximately one hour to complete and was performed in accordance with the ethical standards laid down in the 1991 Declaration of Helsinki.

\subsection{Apparatus and stimuli. Stimuli were produced using TACTOS (Gapenne, Rovira, Ali} Ammar, \& Lenay, 2003), a custom-built device that generates tactile stimuli within a $4 * 4$ matrix design. The $4 * 4$ pin-matrix display measures $2 \mathrm{~cm}$ in width and $2.5 \mathrm{~cm}$ in height. When activated, each pin extends $2 \mathrm{~mm}$ from the display surface. Observers sat with their head approximately $60 \mathrm{~cm}$ from the device, which was Velcro-taped to a stable frame. During the experiment, observers wore an eye mask to stop visual information about the device influencing their judgements. Tactile signals were on/off activations of the Braille pins. The letter stimuli were formed by sequential activations of the pins as shown in Figure 1 . The rate of activation was $20 \mathrm{~mm} / \mathrm{sec}$. The stimulus lasted approximately $1000 \mathrm{~ms}$ in total with each pin activation corresponding to $100 \mathrm{~ms}$. Each letter was defined according to the pattern of stimulation on the device surface. 


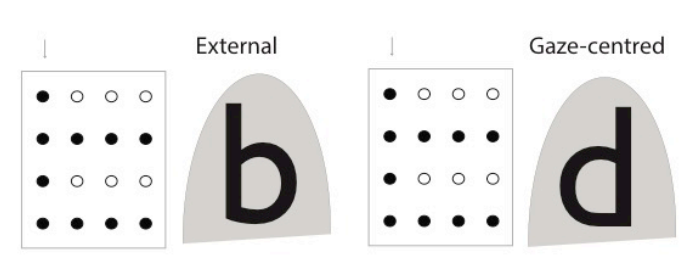

\begin{tabular}{|c|l|l|l|}
\hline Facing away & $\begin{array}{l}\text { Facing away } \\
\text { Perpendicular }\end{array}$ & Facing towards & $\begin{array}{l}\text { Facing towards } \\
\text { Perpendicular }\end{array}$ \\
\hline dd. & & dd & \\
\hline
\end{tabular}

c) Adaptation task to mirror reverse perspective

d) Post-test generalisation across fingers and body midline

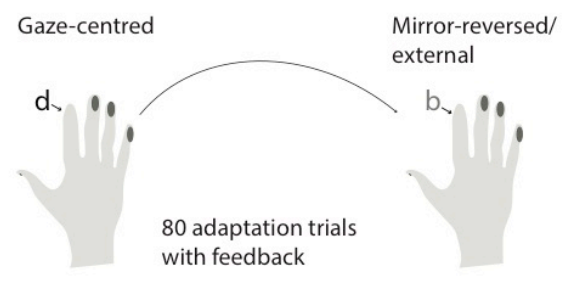

\begin{tabular}{|c|l|l|l|}
\hline $\begin{array}{l}\text { Adapted } \\
\text { index finger }\end{array}$ & $\begin{array}{l}\text { Middle finger } \\
\text { same hand }\end{array}$ & $\begin{array}{l}\text { Index finger } \\
\text { other hand }\end{array}$ & $\begin{array}{l}\text { Middle finger } \\
\text { other hand }\end{array}$ \\
\hline b. & \\
\hline
\end{tabular}

Figure 2. Cutaneous patterns, potential perspectives, and fingertip orientations adopted during the experiment. a) The device pin activation pattern for the letter ' $b$ '. The interpretation of the pattern as a ' $b$ ' required assigning external coordinates to the activation pattern. An interpretation for a ' $d$ ', would involve assigning the reverse, gaze-centred, coordinates. b) The four surface orientations tested during task 1: orientation manipulation. Despite changes in the orientation of the surface relative to the head and the device front/back surface, it is always the volar surfaces of the fingertip that were stimulated. The orientations are given with reference to the head of the observer. c) Illustration of the adaptation phase used in task 2. d) Fingers tested during the postadaptation phase. Orientation of the fingertip was held constant.

\subsection{Procedure. The experiment was divided into three tasks: First, an orientation} manipulation, followed by a verification and an adaptation phase, and finally a post-test generalisation in perspective taking task (see Figure $2 \mathrm{~d}$ ). The first task was divided into four blocks, one for each of the four hand orientations. The participants sat and first fixated a central fixation point on the wall in front of them. They placed the volar surface of their index fingertip, in the orientation indicated by the experimenter, against the device display surface. The fingertip of the dominant hand of the observer was stimulated on the volar surface. The blocked, postural manipulation did not alter the surface stimulated but rather the orientation of the surface relative to the head of the observer and the front/back surface of the device. The participants were then blindfolded to ensure that visual information did not bias their responses. The device was attached to a stable frame so that the participants could press their fingertip against the active surface of the device. Each trial consisted of a single sequential tactile pattern, randomly selected from the stimulus list: 'b', 'd', 'p', or ' $q$ '. Each letter began from the stem and consisted of a continuous motion ending with the body of the letter. We used a four-alternative forced-choice procedure in which the participants 
indicated which of the four letters they perceived, by pressing the corresponding key on the keyboard. The inter-trial interval varied randomly between 1000 and $2000 \mathrm{~ms}$. The participants were able to respond during the stimulus presentation and up to $3000 \mathrm{~ms}$ after stimulus offset. They received no feedback as to the correctness of their responses. Each stimulus was repeated ten times in random order for each blocked hand orientation (i.e. 160 trials in total).

Task 2 consisted of an initial verification of the orientation dependent perspective, then an adaptation phase in which participants adopted orientation 1, as represented in Figure $2 b$, and were asked to reverse the assignment of left/right relative to their responses in the first experiment. That is, they were required to respond as though they were looking at the cutaneous pattern from the perspective of the device. Correct/incorrect feedback was provided over the eighty adaptation trials, with twenty repetitions of each of the four cutaneous letter patterns.

The third task consisted of four post-test blocks, one for each of the generalisation surfaces tested (see Figure $2 \mathrm{~d}$ ). In this phase the strength and generalizability of the adapted perspective was verified. In this post-test phase, the task was identical to that of the first part of the experiment. However, the hand orientation did not change across blocks: the hand was kept in the 'facing away' orientation, but we changed the surface that was stimulated: the adapted index finger surface and three non-adapted finger surfaces (see Figure 2d).

The dominant hand of the observer was used during the pre-test and adaptation phases of the experiment. In the post-test phase, both hands were stimulated. The stimulated region was always the volar surface of the fingertip. During the first two phases of the experiment (i.e., the pre-test and the adaptation phase), the index finger of the dominant hand was used. In the generalisation post-test phase, however, the volar surface of the two index fingers and middle fingers were stimulated, as shown in Figure $2 \mathrm{~d}$.

\section{Results}

The participants' responses were transformed from letter identification estimates to proportions of external or gaze-centred (i.e., mirror-reversed) coordinates. External coordinates were defined as imposing spatial relations onto the pattern of stimulation according to the external device coordinates. In this case, the proportion of responses corresponding to this perspective was calculated as the number of 'b'/'d'/'p'/'q' responses consistent with a 'b'/'d'/'p'/'q' pattern on the device surface. Gaze-centred coordinates were defined as the mirror-reverse of this assignment, with left/right, up/down being assigned 
according to the eyes/head of the participant. Thus, the response observed indicates the perspective adopted by the participant during the tactile stimulation.

For the first task (orientation manipulation), we analysed the proportion of responses consistent with the participants adopting each of the four possible perspectives: external, gaze-centred, external but inverted, and gaze-centred but inverted. A one way repeated measures ANOVA was conducted on the different letter stimuli (4 levels) which failed to reveal any significant difference in the perspective taken across the four letters $[F(3,13)<1$, $p>0.05]$. Consequently, the data were averaged across the different cutaneous letter patterns.

a)

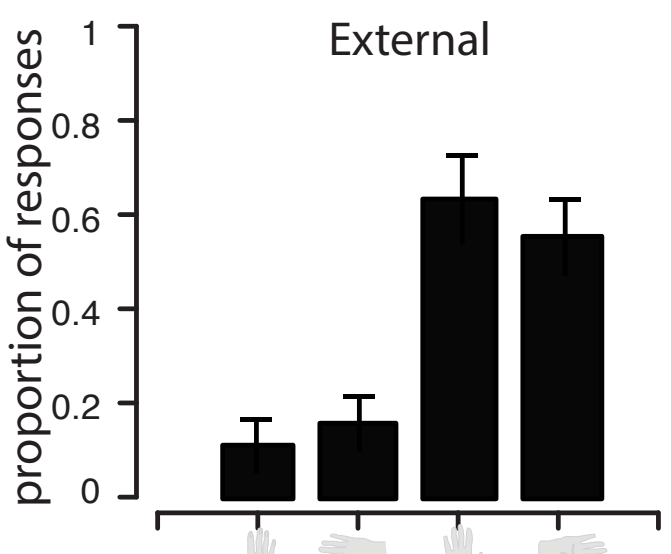

Orientation

c)

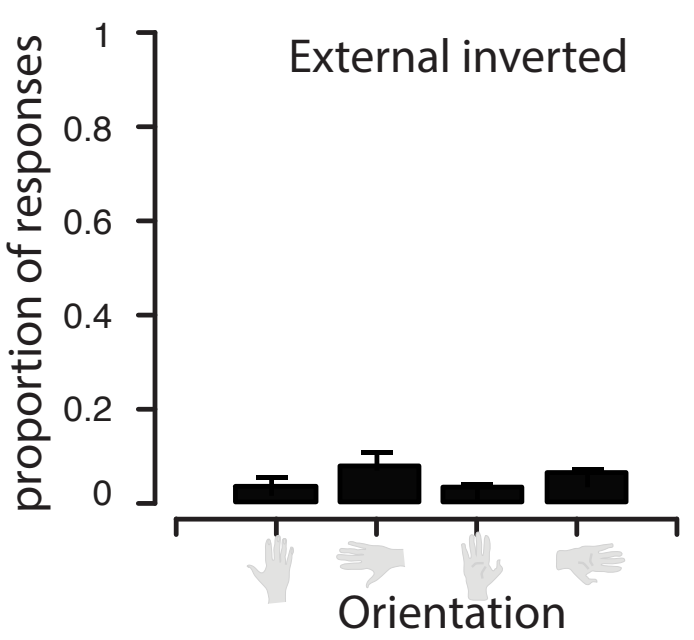

b)

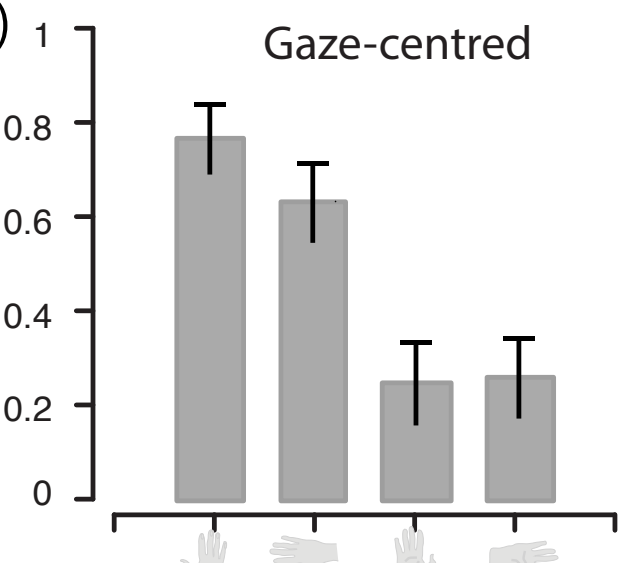

Orientation

d)

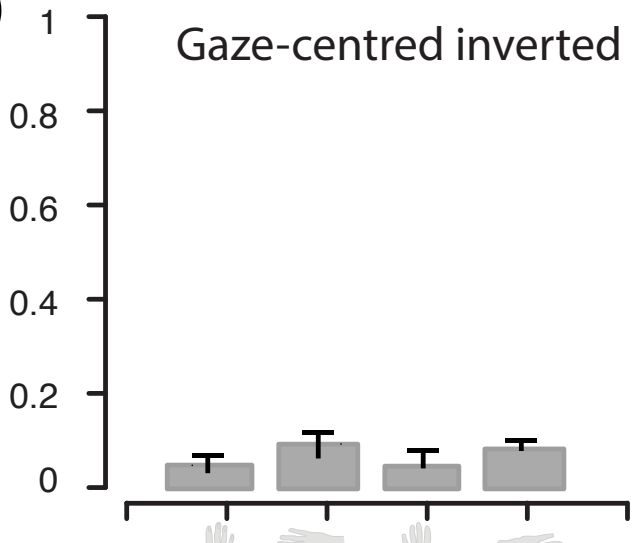

Orientation

Figure 3. The proportion of responses for each orientation, averaged across the four cutaneous patterns 'b', 'd', 'p', and ' $q$ ' consistent with assigning (a) external, (b) gaze-centred, (c) external but horizontally inverted, (d) gaze-centred but horizontally inverted coordinates to the cutaneous pattern. The proportion of responses sum to one across the four possible perspectives. The error bars represent the standard error of the mean across participants.

A repeated measures ANOVA, conducted on the participants' responses with the four-level orientation factor was significant $[F(3,33)=53.22, p<0.001]$. Post-hoc pairwise comparisons with Bonferroni correction showed significant differences between orientations 
1 and 3 (see Figure $2 \mathrm{~b}$ ), [t(26) $=4.37, p=0.001]$ and between orientations 1 and 4 [t(26) $=$ 4.34, $p=0.002]$. There was no difference between orientations 1 and $2[\mathrm{t}(26)=1.15, p=$ $0.25]$, nor between 3 and 4 [t(26) $=0.09, p=0.92$ ]. These results demonstrate that the participants' perspective depends on the orientation of the hand relative to the device with the differences being driven by the facing away versus facing toward positions, the upright versus perpendicular position having no influence on the results (Figure 3a). In other words, the participants' perspective depends on the orientation of their hand along the vertical axis. With respect to the adopted perspective, when the hand is facing toward the participant, both when upright and perpendicular to the body midline, an external perspective is adopted. When the hand is oriented facing away from the participant, a gaze-centred perspective is taken on the tactile patterns of stimulation. The inverted perspectives were adopted significantly less than chance [t(13) $>1, p<0.012]$, see Figure $3 c \& 3 d$.

With respect to task 2, we analysed what happens during the pre-test, the adaptation phase, and the post-test of the experiment, respectively. During the pre-test, the proportion of responses was consistent with the gaze-centred perspective predicted from the results obtained during the pre-adaptation task (see Figure 4a). The gaze-centred perspective was mainly adopted ( $82 \%$ of the trials), and the three other perspectives occurred significantly less often than chance $[\mathrm{t}(11)=-2.49, p=0.95, \mathrm{t}(11)=-20.28, p=1, \mathrm{t}(11)=-16.69, p=0.9]$, see Figure 4a.

During the adaptation task, the perspective is hypothesised to shift from the initial perspective and converge towards the adapted perspective as a function of time (see Figure $4 b)$. Figure $4 b$ represents the proportion of responses consistent with the nonadapted perspective across trials, averaged across the fourteen participants and across the four letters. To understand the adaptation rate for novel perspective taking in touch, we fit a power function to the adaptation data. The function has a slope of $0.8489(95 \% \mathrm{Cl}=$ $0.6912,1.007)$ and an intercept of $-0.409(95 \% \mathrm{Cl}=-0.5315,-0.2864)$, with $\mathrm{R}^{2}=0.7807, p$ $<0.01$. From the fit we can predict that saturation in the amount of adaptation is expected after about 100 adaptation trials where the function reaches a local minimum. The change in response over time during adaptation demonstrates that our feedback (correct/incorrect response) modified the responses as to which letter was felt on the fingertip.

With respect to the generalisation post-test, a repeated measures ANOVA was conducted on the participants' responses with one factor: finger stimulated (4 levels). The analysis did not reveal significant differences in the perspective taken for any of the surfaces tested, the adapted one or the three non-adapted $[F(3,33)=1.938, p=0.14]$. Moreover, single sample t-tests, with Bonferroni correction applied, revealed that the transfer effect is significantly higher than chance $[\mathrm{t}(11)>2.5, p<0.002]$ for all surfaces tested. Thus, the learnt perspective, 
consistent with mapping the stimulation into external coordinates remains active for the adapted finger, and generalises across the non-adapted, middle finger of the same hand and across the body midline to the homologous and middle finger of the non-adapted hand (Figure 4c). These results show that the orientation cues can be overridden by training to the reverse perspective: causing participants to map the cutaneous information into external coordinates.
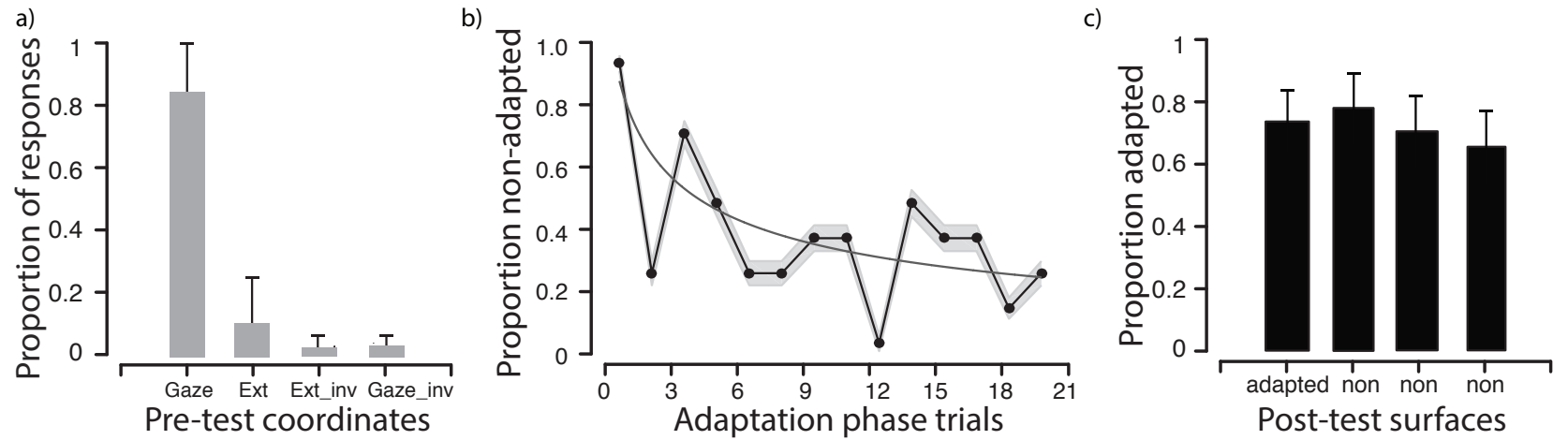

Figure 4. The proportion of responses consistent with the natural and adapted perspectives, before, during, and after the adaptation task. a) The proportion of responses consistent with the predicted, gaze-centred perspective, and the three other non-predicted ones, averaged across observers. The error bars represent the standard error of the mean. b) The change in the response pattern during adaptation (averaged across observers and across the 4 letters). That is, adaptation phase trial 1 corresponds to 4 trials involving each of the four letters $b, d, p, q$ ). The variance (shaded error bar) represents the standard error across observers. The polynomial fit to the proportion non-adapted responses, represented by the continuous grey line. c) The proportion of responses in the post-test (averaged across observers and letters) consistent with the adapted perspective for the four surfaces: the adapted fingertip, the non-adapted middle finger of the adapted hand, the homologous finger of the non-adapted hand, and the middle finger of the non-adapted hand. The error bars represent the standard error of the mean.

\section{Discussion}

Our study explored whether postural cues can account for the mind's perspective on touch, whether the perspective is malleable and can be updated to a novel perspective via adaptation, and finally if adaptation of one surface transfers to non-adapted surfaces.

\subsection{Orientation}

The first main result to emerge from our study is that the perspective taken on ambiguous tactile patterns presented to the fingertip is dependent on the posture of the hand, i.e., its orientation. The results show a dependence on orientation around the vertical axis, a rotation of 90 from the vertical to the horizontal axis did not influence the results. 
Parsons and Shimojo (1987) previously explored whether the perspective taken on stimulation of the palm and dorsal regions of the hand is affected by orientation of the hand. Observers in their study adopted seven different hand orientations. For orientations in which the hand was in front of them, the observers interpreted the cutaneous pattern primarily according to external coordinates for both surfaces of the hand. These results are consistent with interpreting the cutaneous pattern according to the experimenter's viewpoint. However, as was outlined in the introduction, in Parsons and Shimojo's experiment, the fact that the experimenter manually traced the letter on the participant's body surface might have biased their responses toward the experimenter's point of view. This would appear to be the case, given that in our study we have removed this potential bias by using an automatized presentation of the stimuli and our results reveal that when the hand is in front of the observer, tactile information is transformed both into external coordinates and into gazecentred coordinates.

With respect to the two coordinate systems that we observed, it should be noted that it is not unusual that touch on the hand is coded into coordinates other than hand-centred. Indeed, viewpoint selection in touch is a non-straightforward process because not only are there multiple different body surfaces, but the position and orientation of the surface relative to the rest of the body, the eyes, and the object itself, strongly influences the reference frame adopted (Parsons \& Shimojo, 1987; Volcic et al., 2009). This leads to the interdependence of several different reference frames, namely those based on somatosensory/body, and external world coordinates (Harrar \& Harris, 2009). Several studies have suggested that tactile information on different surfaces can also be remapped into a gaze-centred, or a motor coordinate system (Harrar \& Harris, 2009; Mancini \& Haggard, 2014). In addition to the multiple potential coordinate systems available for coding touch, we assumed that the stimulation on the fingertip may be influenced further by the fact that the fingers can move independently to the rest of the hand (functionally driven) and may therefore recruit additional coordinate systems for decoding stimulation, to that used for interpreting touch to the rest of the hand.

\subsection{Adaptation}

The second result to emerge from our study concerns the plasticity in the perspective taken as a result of adaptation. When the participants held the orientation of their hand constant and were adapted to a mirror-reversed perspective, as compared with their initial choice for that orientation in task 1 , the interpretation of the ambiguous cutaneous patterns was updated to the adapted (external) perspective. The results of this adaptation phase demonstrate that the mind's perspective on touch is malleable and plastic in the face of 
feedback. Note that the adaptation to the reverse perspective occurred rapidly, during the eighty trials of adaptation (20 for each cutaneous pattern). The fact that simple correctincorrect feedback can override the low-level postural cue of orientation supports the idea that there is already an ambiguity in terms of which coordinate system might be applied to the pattern of stimulation on the skin and given that the brain must resolve an existing conflict between multiple possible coordinate assignments for touch, a higher-order learning factor can therefore easily override low-level cues (Corcoran, 1977; Parsons \& Shimojo, 1987; Volcic et al., 2009).

\subsection{Transfer of learning}

The results from the third task of our study demonstrate that the adapted perspective transfers across non-adapted fingers and across the body midline (i.e., the other hand). This, in conjunction with the fact that 'correct'/'incorrect' feedback can mediate the adopted perspective (overriding orientation cues), suggest that the process we measured here is a higher-level perspective taking, rather than an automatic coordinate assignment process linked to the receptive fields of the stimulated surfaces (Harrar, Spence, \& Makin, 2014).The strength of the transfer did not decrease significantly when tested on non-adapted surfaces, which is interesting because it suggests that the adapted perspective relies on something more than perceptual factors (Spengler et al., 1997). Moreover RT data (see supplementary material) suggest that the process we observe is not solely the result of mental rotation, given that the difference between RT data in the pre- and post-test phases is only approximately $8 \mathrm{~ms}$. While significant, this is a different order of magnitude to that observed in mental rotation tasks (see for example, Just \& Carpenter, 1985).

For learning of tactile patterns on different body surfaces, i.e., the trunk and leg of the observer, Arnold and Auvray (2014) have also found a transfer of learning to non-adapted surfaces. The authors suggest that their effect is driven by the organisation of the somatosensory cortex. However, in our case where the transfer is about reference frame rather than patterns of stimuli, the fact that the learning transfers across the body midline suggests that the transfer at stake occurs at later stages in the processing pathway, given the lateralisation of early somatosensory processing (Boven, Ingeholm, Beauchamp, Bikle, \& Ungerleider, 2005). The ability to train a person to adopt the mirror reverse perspective may be due to the fact that the stimuli themselves are high level and therefore recruit higher order processing regions.

The transfer results have implications for training with novel haptic interfaces, suggesting that it is possible to remap, with a limited amount of training, the local cutaneous pattern of 
stimulation into different coordinates for the purposes of experiencing a three dimensional world and a novel perspective from your own gaze/body-centred point of view.

\subsection{Implications of the results}

For the results obtained across the three tasks of our study: the perspective on touch adopted as a function of hand orientation, adaptation, and transfer, we could speculate that a similar level of processing is actually involved, given that the cutaneous patterns were not found to be coded in hand-centred coordinates for any orientations. That is, our results can be explained by higher order processes probably involving brain areas not organised topographically (see also Harrar et al., 2014). It is clear that the observer's interpretation of patterns of stimulation on the skin is influenced by a number of factors, including competing coordinates systems (Parsons \& Shimojo, 1987), object identity (G. Arnold \& Auvray, 2014), the goal of the observer (Oldfield \& Phillips, 1983), and the observer's natural preferences (Gabriel Arnold, Spence, \& Auvray, 2016). What we observe with passive stimulation of tactile letters is that the competition between these different factors is resolved by stable high-level factors. We can quantify the perspective at the fingertip as being driven by postural cues but informed ultimately by learning, mental rotation, and feedback.

This can be accounted for by the fact that in order to maintain a stable experience of our environment, despite our ability to move our sensors relative to the environment, we need to be able to take into account the change in our own sensor positions (Hartcher-O'Brien \& Auvray, 2014). Thus, the orientation dependence observed in the first task of the current study highlights one way in which the brain tries to account for changes in sensor position; i.e., to map information into gaze-centred and external coordinates. For visual processing, the visual system remaps information into external coordinates in order to maintain a stable representation across eye and stimulus movements (Epstein, 1977). Here our study shows how the brain updates its perspective on cutaneous stimulation during changes in hand orientation, transforming the patterns into non-hand based coordinates. That is, in order to maintain a stable representation of the environment, the brain selects predominantly gazecentred and external coordinates during the processing of passive tactile information.

To conclude, the co-ordinate system chosen to interpret tactile objects is a complex interplay between the object position in space, on the skin, and the orientation of the body part receiving the stimulation relative to other body surfaces (Parsons \& Shimojo, 1987). In our study, in no case was the hand-centred perspective adopted over the external and gazecentred perspectives. The importance of hand orientation in perspective taking in everyday life, as demonstrated in the first part of the experiment, is highlighted in the example of how your brain uses the orientation of your hand to infer the surface (top or bottom) of a table: 
When you rest your hand on the top of a table, your perspective on the table's surface is unambiguous and primarily coded from the orientation of your hand. You know that your hand is in contact with the table-top. If you rotate your hand 180 degrees and establish contact with the table again, it is clear that you are in contact with the lower surface of the table. In any construction of external objects and space, it is important to provide coordinates that can be used to determine the spatial relations among objects and our senses. When we touch objects or when we are touched, both physical and physiological constraints influence our interpretation of the objects we perceive. Nevertheless, cognitive factors can override physiological constraints as seen in the adaptation of the mind's perspective on touch. 


\section{References}

Arnold, G., \& Auvray, M. (2014). Perceptual learning: Tactile letter recognition transfers across body surfaces. Multisensory Research, 27(1), 71-90. http://doi.org/10.1163/22134808-00002443

Arnold, G., Spence, C., \& Auvray, M. (2016). Taking someone else's spatial perspective: Natural stance or effortful decentring? Cognition, 148, 27-33. http://doi.org/10.1016/j.cognition.2015.12.006

Azañón, E., \& Soto-Faraco, S. (2008). Changing Reference Frames during the Encoding of Tactile Events. Current Biology, 18(14), 1044-1049.

Boven, R. W. V., Ingeholm, J. E., Beauchamp, M. S., Bikle, P. C., \& Ungerleider, L. G. (2005). Tactile form and location processing in the human brain. Proceedings of the National Academy of Sciences of the United States of America, 102(35), 12601-12605. http://doi.org/10.1073/pnas.0505907102

Corcoran, D. W. J. (1977). The Phenomena of the Disembodied Eye or is it a Matter of Personal Geography? Perception, 6(3), 247-253. http://doi.org/10.1068/p060247

Epstein, W. (1977). Stability and constancy in visual perception: Mechanisms and processes. New York, NY, USA: John Wiley \& Sons.

Farrell, M. J., \& Robertson, I. H. (1998). Mental rotation and automatic updating of body-centered spatial relationships. Journal of Experimental Psychology: Learning, Memory, and Cognition, 24(1), 227-233. http://doi.org/10.1037/0278-7393.24.1.227

Gapenne, O., Rovira, K., Ali Ammar, A., \& Lenay, C. (2003). Tactos: Special computer interface for the reading and writing of 2D forms in blind people. Universal Access in HCl, Inclusive Design in the Information Society, 10, $1270-1274$. 
Harrar, V., \& Harris, L. (2009). Eye position affects the perceived location of touch. Experimental Brain Research, 198(2-3), 403-410. http://doi.org/10.1007/s00221-009-1884-4

Harrar, V., Spence, C. J., \& Makin, T. R. (2014). Topographic generalization of tactile perceptual learning. Journal of Experimental Psychology: Human Perception and Performance, 40(1), 15-23. http://doi.org/10.1037/a0033200

Hartcher-O'Brien, J., \& Auvray, M. (2014). The process of distal sttribution illuminated through studies of sensory substitution. Multisensory Research, 27(5-6), 421-441.

Heed, T., Buchholz, V. N., Engel, A. K., \& Röder, B. (2015). Tactile remapping: from coordinate transformation to integration in sensorimotor processing. Trends in Cognitive Sciences, 19(5), 251-258. http://doi.org/10.1016/j.tics.2015.03.001

Holmes, N. P. (2014). Hand-centred space, hand-centred attention, and the control. Cortex, 25, 189-220.

Holway, A. H., \& Boring, E. G. (1941). Determinants of apparent visual size with distance variant. The American Journal of Psychology, 54(1), 21-37.

Just, M. A., \& Carpenter, P. A. (1985). Cognitive coordinate systems: Accounts of mental rotation and individual differences in spatial ability. Psychological Review, 92(2), 137-172. http://doi.org/10.1037/0033-295X.92.2.137

Mancini, F., \& Haggard, P. (2014). Perception: A Motion After-Effect for Voluntary Actions. Current Biology, 24(2), R70-R72.

Newell, F. N., Ernst, M. O., Tjan, B. S., \& Bülthoff, H. H. (2001). Viewpoint dependence in visual and haptic object recognition. Psychological Science, 12(1), 37-42.

Oldfield, S. R., \& Phillips, J. R. (1983). The Spatial Characteristics of Tactile Form Perception. Perception, 12(5), 615-626. http://doi.org/10.1068/p120615 
Parsons, L., \& Shimojo, S. (1987). Perceived spatial organization of cutaneous patterns on surfaces of the human body in various positions. Journal of Experimental Psychology: Human Perception and Performance, 488-504.

Prather, S. C., \& Sathian, K. (2002). Mental rotation of tactile stimuli. Cognitive Brain Research, 14(1), 91-98.

Schicke, T., \& Röder, B. (2006). Spatial remapping of touch: Confusion of perceived stimulus order across hand and foot. Proceedings of the National Academy of Sciences, 103(31), 11808-11813. http://doi.org/10.1073/pnas.0601486103

Spengler, F., P.L. Roberts, T., Poeppel, D., Byl, N., Wang, X., Rowley, H. A., \& Merzenich, M. M. (1997). Learning transfer and neuronal plasticity in humans trained in tactile discrimination. Neuroscience Letters, 232(3), 151-154. http://doi.org/10.1016/S0304-3940(97)00602-2

Torralbo, A., Santiago, J., \& Lupiáñez, J. (2006). Flexible conceptual projection of time onto spatial frames of reference. Cognitive Science, 30(4), 745-757. http://doi.org/10.1207/s15516709cog0000_67

Vallbo, A. B., \& Johansson, R. S. (1984). Properties of cutaneous mechanoreceptors in the human hand related to touch sensation. Human Neurobiology, 3(1), 314.

Volcic, R., Wijntjes, M. W. A., \& Kappers, A. M. L. (2009). Haptic mental rotation revisited: multiple reference frame dependence. Acta Psychologica, 130(3), $251-259$.

Wexler, M., \& Held, R. M. (2005). Anticipating the three-dimensional consequences of eye movements. Proceedings of the National Academy of Sciences of the United States of America, 102(4), 1246-1251. 